\title{
The Human Element of Breast Cancer: Insights from Pakistan
}

\section{Maggi Banning*}

School of Health Sciences and Social Care, Brunel University, West London, England

Pakistan is a developing country that has the highest rate of breast cancer in Asia [1]. This is reflected in the age standardised ratio is 69.1 per 100,000 averaged from $1998-2002$ [2]. Women as young as aged 21 years develop breast cancer [3]. This is possibly the youngest reported case of breast cancer in Asia. In Pakistan, women of young age also present at with advanced and aggressive forms of breast cancer which negatively impact on prognosis $[3,4]$. Reasons for the delay in seeking treatment may relate to the negative social-cultural perception of cancer and inherent fear of families response to cancer $[5,6]$. It is also due to a fear of an unknown, unrecognised disease that women may not have heard of before as a result of no family history. Studies show that women may seek advice from spiritual peers, or purchase traditional medicines in an attempt to treat the illness, rather than seeking medical advice [6]. The grief, anxiety and often depression that women feel can be accompanied by, or the result of the family reaction to the disease. In Pakistan, cancer is perceived as a contagious illness. It is a disease that is not discussed even among female members. Through education, women may be aware of breast cancer screening practices. "I think that women in our society are told from a very young age that this organ is to be kept secret, so they don't talk about it very openly with their parents or to their relatives unless they talk about it to their friends only. The disease is hidden. That's why they are diagnosed at fourth stage when there is no option but surgery" [7].

For many women, a diagnosis of breast cancer may led to feelings of social isolation from family members and also friends who distance themselves from women due to their condition and its perceived impact on their own health. This impact has been reported in women with both early stage and advanced breast cancer $[4,7,8]$. Many women feel isolated and this can be not only detrimental to the ability of an individual to adjust to the disease but also survival rates [9]: "I feel that I should die when I realise the treatment process but when I see my children, I want to live. I am worried about my children. I came here with my husband and left my children alone, as the village people don't care about my children in case they get the disease. Family members live far away, they don't eat with us as they think they think will get the same disease if they meet me, that is why I am alone" [4].

Once families agree that their wife or female family member require medical assistance to treat their cancer, two options are available to them. Women can either attend a local cancer unit within a Government hospital, or a specialist cancer hospital where the treatment is free. The treatment offered will be prescribed by a medical physician and implemented via a care plan by the nurses on the ward. Families have to purchase nursing supplies such as intravenous fluids, injections, wound dressings. The patients' family also have to provide the patient with fluids and meals. Due to high patient demand, hospital wards tend to be overcrowded with deficient supply of qualified of nurses. Nursing care is commonly provided by nursing students who are often overworked with limited nursing experience. The pressure of high patient/ nurse ratio, one nurse to 90 patients and extensive patient demands results in variable quality of patient care and a situation where patient empathy, education, counselling and the provision of indivualised care may be lacking. In such cases, the humane aspect of how the patient handles the emotional effects of cancer may not be acknowledged. Women continually live with a fear of the unknown, managing the in-depth sorrow related to the diagnosis but also the emotional reaction to chemotherapy that women cite usually occurs three days following the completion of chemotherapy [6]. Such reactions can result in anxiety, tearfulness, depression and trepidation related to possible cancer recurrence and fear related to the future of their children $[6,7]$. In Government hospitals, nursing staff may not be trained in the provision of patient education, the development of, and awareness of, the importance of patient counselling and its impact on the holistic care that a patient should receive [4]. Nurses may not be sensitive to the needs of patients and their families due to the high numbers of patients receiving care simultaneously.

In comparison, families that can afford to finance private treatment for breast cancer, or economically disadvantaged families can apply for free cycles of chemotherapy treatment and treatment provided by Shaukat Khnanum Cancer Memorial Hospital. In this hospital, medical and nursing staff are trained oncology specialists. Nurses undertake specialist post registration education and training in oncology care. The philosophy of the nursing department is to provide holistic care to cancer patients and their families. A key function of patient care is to provide health education, counselling and empathic care to patients they care for [9]. Many of the patients attend the hospital on a routine basis for repeated cycles of chemotherapy treatment, so patients become familiar with the nurses that care for them. Many patients reveal their anxiety, sorrow and trepidation regarding their illness and fear of recurrence, in response nurses counsel and educate patients and their families from an oncology perspective but also from experience [9]. In this way, patients and their families appreciate the educational and emotional support provided by nurses and medical staff. As a result of the continuous commitment to holistic patient care, the hospital has developed a reputation for excellence in medical and nursing patient care. The ethos of the nursing department is to put the patient first and to be sensitive to patients unique circumstances.

\section{References}

1. Jemal A1, Bray F, Center MM, Ferlay J, Ward E, et al. (2011) Global cancer statistics. CA Cancer J Clin 61: 69-90.

2. Bhurgri Y1, Bhurgri A, Nishter S, Ahmed A, Usman A, et al. (2006) Pakistancountry profile of cancer and cancer control 1995-2004. J Pak Med Assoc 56 124-130.

3. Badar F1, Moid I, Waheed F, Zaidi A, Naqvi B, et al. (2005) Variables associated with recurrence in breast cancer patients-the Shaukat Khanum Memoria experience. Asian Pac J Cancer Prev 6: 54-57.

4. Banning M1, Tanzeen T (2014) Living with advanced breast cancer: perceptions of Pakistani women on life expectations and fears. Cancer Nurs 37: E12-18.

5. Banning M1, Hassan M, Faisal S, Hafeez H (2010) Cultural interrelationships and the lived experience of Pakistani breast cancer patients. Eur J Oncol Nurs 14: 304-309.

${ }^{*}$ Corresponding author: Dr. Maggi Banning, School of Health Sciences and Social Care, Brunel University, West London, England, Tel; 018952 68828; E-mail: maggi.banning@brunel.ac.uk.

Received February 12, 2014; Accepted March 30, 2014; Published April 02, 2014

Citation: Banning M (2014) The Human Element of Breast Cancer: Insights from Pakistan. Primary Health Care 4: 154. doi:10.4172/2167-1079.1000154

Copyright: (c) 2014 Banning M. This is an open-access article distributed under the terms of the Creative Commons Attribution License, which permits unrestricted use, distribution, and reproduction in any medium, provided the original author and source are credited. 
6. Banning M1, Hafeez H, Faisal S, Hassan M, Zafar A (2009) The impact of culture and sociological and psychological issues on Muslim patients with breast cancer in Pakistan. Cancer Nurs 32: 317-324.

7. Banning M1, Hafeez H (2010) A two-center study of Muslim women's views of breast cancer and breast health practices in Pakistan and the UK. J Cancer Educ 25: 349-353.
8. Banning M1, Hafeez $\mathrm{H}$ (2009) Perceptions of breast health practices in Pakistani Muslim women. Asian Pac J Cancer Prev 10: 841-847.

9. Banning M1, Gumley VA (2012) Clinical nurses' expressions of the emotions related to caring and coping with cancer patients in Pakistan: a qualitative study. Eur J Cancer Care (Engl) 21: 800-808. 